\title{
Evaluación de la fertilidad química del suelo en un sistema inicial productivo de Theobroma cacao de la finca "Hermanos Briones", Portoviejo - Ecuador
}

\begin{abstract}
(c) $\underset{\mathrm{BY} \text { (i) (2) }}{\mathrm{SA}}$
\end{abstract}
Evaluation of the chemical fertility of the soil in an initial productive Theobroma cacao system of the "Hermanos Briones" farm, Portoviejo Ecuador

Javier Ignacio Briones García. ${ }^{1}$, Teresita Jackelin Mejía Reinoso. ${ }^{2} \&$ Huber David
Briones García. ${ }^{3}$

Recibido: 23-06-2021 / Revisado: 30-06-2021 /Aceptado: 20-07-2021/ Publicado: 05-08-2021

\begin{abstract}
. https://doi.org/10.33262/concienciadigital.v4i3.1.1825

Introduction. Soil is the most important natural resource and most of life on earth depends on it, its chemical composition is constantly changing, resulting in countless soils. The use of N, P, K, Ca and Mg fertilizers has become essential in most soils in order to obtain high yields and a good quality in the product, being essential to constantly monitor the soil where the crops are grown. The yields in cacao production depend on the soil and certain environmental factors such as light, temperature and humidity. Flattering yields cannot be achieved when, despite the adequate environment, the services provided by the soil are not adequate (Pinargote, 2010). Objective. To evaluate the chemical fertility of the soil in initial productive system of Theobroma cacao, in the "Hermanos Briones" farm in the Portoviejo canton. Methodology. The research work was carried out in an agricultural batch of an initial 2-year cacao crop of 2.4 ha, the cacao plantation used corresponds to the $\mathrm{CCN}-51$ clone, the soil samplings were carried out at random in a

1 Escuela Superior Politécnica de Chimborazo, Sede Morona Santiago, Morona Santiago, javier.briones@espoch.edu.ec, https://orcid.org/0000-0002-2675-3495

2 Escuela Superior Politécnica de Chimborazo, Maestría en Ingeniería Química Aplicada, Chimborazo, jmejiareinoso@yahoo.com, https://orcid.org/0000-0003-0432-1293

3 Universidad Técnica de Manabí, Ingeniería Eléctrica, Estudiante de pregrado, Manabí, brionesgarciah@gmail.com
\end{abstract}


systematic way (zig-zag ), obtaining a composite sample, to carry out the chemical analyzes in the laboratory, given analyzes were carried out prior to planting and 2 years after cultivation. Results. The comparison of the nutritional content of the soil is observed, with respect to organic matter there is an increase as the plantation grows, the development of organic matter is greater. The macronutrients Nitrogen and Potassium show a slight decrease, contrary to Phosphorus, the mesonutrients Calcium, Magnesium and Sulfur are in smaller quantities, the micronutrients Zinc and Copper show an increase, different from Iron and Boron, which after 2 years of seedlings have decreased the concentrations of these elements in the soil, with respect to Manganese the concentration has not changed. Conclusions. According to the results of the sampling, the initial productive system presents nutritional imbalances and deficiencies in nutrient content for its development, which is why fertilization plans are required to guarantee adequate sustainability of the system.

Keywords: Fertility, chemical analysis, soil, cacao.

\section{Resumen.}

Introducción. El suelo es el recurso natural más importante y la mayor parte de la vida en la tierra depende de este, su composición química varia constantemente, dando como resultado un sinnúmero de suelos. El uso de fertilizantes $\mathrm{N}, \mathrm{P}, \mathrm{K}, \mathrm{Ca}$ y $\mathrm{Mg}$ se ha vuelto indispensable en la mayoría de suelos con el fin de obtener altos rendimientos y una buena calidad en el producto siendo indispensable monitorear constantemente el suelo en donde se desarrollan los cultivos. Los rendimientos en la producción de cacao dependen del suelo y de ciertos factores del medio ambiente como son luminosidad, temperatura y humedad. No podrán alcanzarse rendimientos halagadores cuando a pesar del ambiente adecuado los servicios que proporcione el suelo no sean los adecuados (Pinargote, 2010). Objetivo. Evaluar la fertilidad química del suelo en este sistema inicial productivo de Theobroma cacao, en la finca "Hermanos Briones" en el cantón Portoviejo. Metodología. El trabajo de investigación se realizó en un lote agrícola de un cultivo inicial de cacao de 2 años de 2.4 ha, la plantación de cacao utilizada corresponde al clon CCN-51, los muestreos del suelo se realizaron al azar en forma sistemática (zig-zag), obteniendo una muestra compuesta, para realizar los análisis químicos en laboratorio, dados análisis se realizaron previo a la plantación y a los 2 años del cultivo. Resultados. Se observa la comparación del contenido nutricional del suelo, con respecto a la materia orgánica existe un incremento conforme va creciendo la plantación el desarrollo de materia orgánica es mayor. Los macronutrientes Nitrógeno y Potasio presentan un ligero descenso lo contrario al Fosforo, los mesonutrientes Calcio, Magnesio y Azufre se encuentran en menores cantidades, los micronutrientes Zinc y Cobre presentan un aumento, diferente al Hierro y Boro que a los 2 años de las plántulas ha disminuido las concentraciones de estos elementos en el suelo, con respecto al Manganeso la concentración no ha variado. Conclusiones. Según los resultados del muestreo, el sistema inicial productivo presenta desbalances nutricionales y deficiencias en contenidos de nutrientes para su desarrollo, por lo cual se requiere de planes de 
fertilización que garanticen una adecuada sostenibilidad del sistema.

Palabras claves: Fertilidad, anàlisis químico, suelo, cacao.

\section{Introducción.}

Según el Resultados nacionales sobre el III Censo Nacional Agropecuario, en el Ecuador existen aproximadamente 400000 has cultivadas de cacao (Theobroma cacao L.), su producción cubre el 60\% del mercado de exportación mundial, solo en el año 2010 está alcanzó más de 173300 TM., de las cuales el 80\% correspondieron a cacao fino y el 20\% al clon CCN-51 (Ministerio de Agricultura y Ganadería, 2014).

Los rendimientos en la producción de cacao dependen del suelo y de ciertos factores del medio ambiente como son luminosidad, temperatura y humedad. No podrán alcanzarse rendimientos halagadores cuando a pesar del ambiente adecuado los servicios que proporcione el suelo no sean los adecuados (Pinargote, 2010). El suelo es el recurso natural más importante y la mayor parte de la vida en la tierra depende de este de forma directa o indirecta, y su composición química varia constantemente, dando como resultado un sinnúmero de suelos. El uso de fertilizantes $\mathrm{N}, \mathrm{P}, \mathrm{K}, \mathrm{Ca}$ y $\mathrm{Mg}$ se ha vuelto indispensable en la mayoría de suelos con el fin de obtener altos rendimientos y una buena calidad en el producto siendo indispensable monitorear constantemente el suelo en donde se va a realizar el cultivo.

Todo buen agricultor debe llevar un registro de las composiciones químicas del suelo en uso, antes de la producción durante y al final, de esta manera estará realizando una contabilidad precisa de los nutrientes que se han removido y reemplazado en el suelo, y de esta manera el productor sabrá aplicar los fertilizantes necesarios para obtener buenas cosechas y mantener un suelo saludable.

Por lo tanto, es importante evaluar la fertilidad química del suelo en este sistema inicial productivo de Theobroma cacao, en la finca "Hermanos Briones" en el cantón Portoviejo, para poder tener una estadística de como el suelo va cambiando a lo largo de su uso en la producción de cacao, de esta manera se podrá realizar recomendaciones que permitan mejorar el manejo y la sostenibilidad de los suelos.

\section{Metodologia.}

\section{Descripción del sitio y muestreo}

El trabajo de investigación se realizó en un lote agrícola de un cultivo inicial de cacao de 2 años de 2.4 ha, la plantación de cacao utilizada corresponde al clon CCN-51 sembrado a $3 \mathrm{~m}$ entre plantas y $3 \mathrm{~m}$ entre surcos, para una densidad de 1.112 plantas por hectárea, las aplicaciones de fertilizaciones edáficas anuales son de 2, mientras que las foliares son cada 4 meses, el sistema de regio en temporal de verano es por aspersión cada 20 días con aguas subterráneas, situado a 15'47.963" LS, 80²3'19.179” LW, en la ciudad de 
Portoviejo - Ecuador, con altitud de 100 ms.n.m., la geología de la zona es variada, pero predomina las calizas, con una clase textural arcillo - limoso, 13\% arena, 44\% limo y 43\% arcilla, en un clima trópico seco - semiárido.

El lote presenta una historia agrícola de alrededor de 40 años, los cultivos predominantes antes de la plantación de cacao fueron: maíz, maní y verdeos invernales para forraje, previo a la plantación del cultivo de cacao el lote registra 15 años de descanso de actividad agrícola.

Los muestreos del suelo se realizaron al azar en forma sistemática (zig-zag), obteniendo una muestra compuesta, por la extracción de varias muestras simples o submuestras, recolectadas de una profundidad 0 a $30 \mathrm{~cm}$, con una barrena, la muestra fue reducida a 1 $\mathrm{kg}$. El suelo se secó a temperatura ambiente, se molió y tamizó en una malla de $2 \mathrm{~mm}$, para realizar los análisis químicos en laboratorio (Sosa, 2012), dados análisis se realizaron previo a la plantación y a los 2 años del cultivo de cacao.

\section{Determinaciones químicas}

Las muestras de suelo se llevaron a laboratorio y se determinó: pH por potenciometría (relación suelo-agua 1:2,5), materia orgánica (MO) por titulación de Walkey Black, el N, $\mathrm{P}$ y B por colorimetría, el S por turbimetria utilizando el fosfato de calcio monobásico como extractante, mientras que la metodología utilizada para determinar el $\mathrm{K}, \mathrm{Ca}, \mathrm{Mg}$, $\mathrm{Cu}, \mathrm{Fe}, \mathrm{Mn}$ y $\mathrm{Zn}$ fue de absorción atómica, de acuerdo a los protocolos establecidos en dicho laboratorio.

\section{Resultados.}

El potencial de hidrogeno $(\mathrm{pH})$ es una de las más importantes determinaciones del suelo, puede afectar directamente la disponibilidad de nutrientes del suelo y la actividad microbiana provocando cambios fisiológicos y bioquímicos en el metabolismo de las plantas (Osorio, 2012).

En la figura 1, se observa una comparación del parametro $\mathrm{pH}$ en el lote de cultivo de cacao, el cual fué de 7.30 previo a la plantación y de 8.00 a los 2 años del cultivo, interpretando los resultados se observa que previo a la plantación del cacao, el suelo contaba con un $\mathrm{pH}$ neutro, a los 2 años de plantación existe un incremento tornando el suelo ligeramente alcalino.

Dada elevación de pH según la entrevista realizada y con fundamentos del autor (Lamz \& González, 2010) es el resultado del uso de aguas salinas subterráneas para el riego de la plantación, lo cual permite la movilidad de las sales dentro del suelo y el transporte de las mismas, además de las condiciones climáticas, déficit de materia orgánica y el tipo de suelo. 


\section{Figura 1}

Comparación del pH del suelo previo a la plantación de cacao \& 2 años de cultivo

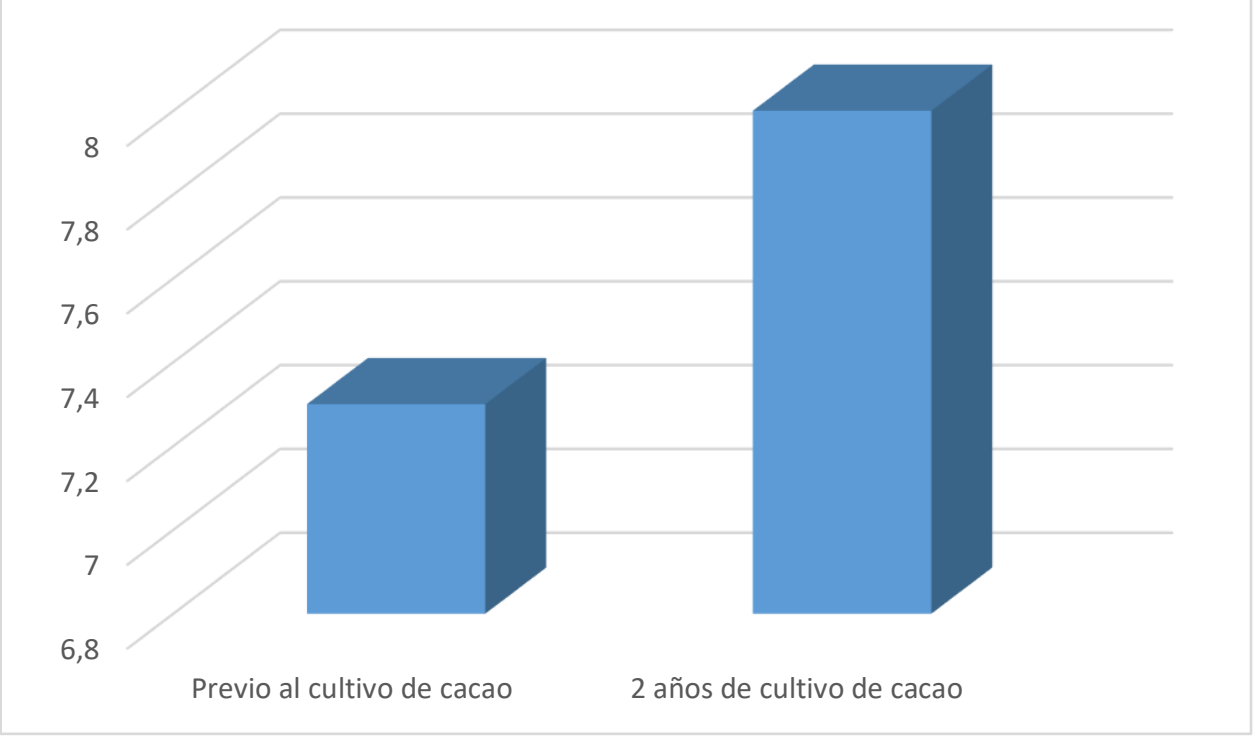

Fuente: Análisis químicos de suelo

Elaborado: por los autores

Según (Arvelo Sánchez et al., 2017; Quiroz \& Mestanza, 2012) recomiendan suelos con pH entre 6.0 y 7.0 para el cultivo de cacao. Este es susceptible a suelos alcalinos y calcareos, cuando hay carbonatos mayores de $6 \%$ la clorosis es severa, su crecimiento es débil, las plantas adultas presentan deficiencias de Hierro, desbalance nutricional, toxicidad ion específica y la producción es baja.

En la tabla 1 se encuentra los resultados de los análisis químicos de suelo realizado al lote, la interpretación se realizó según los criterios establecidos por (Amésquita et al., 2001), previo a la plantación de cacao, se observa bajo porcentaje de materia orgánica (M.O,), y Manganeso (Mn), niveles medios de Nitrógeno Amoniacal (NH4), Magnesio $(\mathrm{Mg})$, Azufre (S), Zinc (Zn), Hierro (Fe) y Boro (B), mientras que los niveles de Potasio $(\mathrm{K})$, Calcio $(\mathrm{Ca})$ y Cobre $(\mathrm{Cu})$ son altos, los niveles de Fosforo $(\mathrm{P})$ son óptimos. A los 2 años de plantación de cacao, contiene bajo porcentaje de materia orgánica (M.O.), Azufre (S), Manganeso ( $\mathrm{Mn}$ ), Hierro (Fe) y Boro (B), niveles medios de Nitrógeno Amoniacal (NH4), Fosforo (P) y Zinc (Zn), mientras que los niveles de Potasio (K), Calcio (Ca), Magnesio $(\mathrm{Mg})$ y Cobre $(\mathrm{Cu})$ son altos.

Tabla 1

Contenido nutricional del suelo previo a la plantación del cacao \& A los 2 años de plantación del cultivo de cacao

$\begin{array}{ccc}\text { Parámetro } & \text { Unidad } & \text { Contenido nutricional del } \\ & \text { suelo previo a la plantación } & \text { Contenido nutricional del } \\ \text { del cacao } & \text { suelo a los } 2 \text { años de } \\ \text { plantación del cultivo de }\end{array}$

cacao

$\begin{array}{llll}\text { M.O. } & \% & 1.2 & 1.7\end{array}$




\section{Tabla 1}

Contenido nutricional del suelo previo a la plantación del cacao \& A los 2 años de plantación del cultivo de caca (continuación)

\begin{tabular}{|c|c|c|c|}
\hline Parámetro & Unidad & $\begin{array}{c}\text { Contenido nutricional del } \\
\text { suelo previo a la plantación } \\
\text { del cacao }\end{array}$ & $\begin{array}{l}\text { Contenido nutricional de } \\
\text { suelo a los } 2 \text { años de } \\
\text { plantación del cultivo de } \\
\text { cacao }\end{array}$ \\
\hline NH4 & Ppm & 30 & 22 \\
\hline $\mathbf{P}$ & ppm & 19 & 20 \\
\hline K & $\mathrm{meq} / 100 \mathrm{ml}$ & 1.15 & 1.01 \\
\hline $\mathrm{Ca}$ & $\mathrm{meq} / 100 \mathrm{ml}$ & 17 & 13 \\
\hline $\mathrm{Mg}$ & $\mathrm{meq} / 100 \mathrm{ml}$ & 4.5 & 2.1 \\
\hline$S$ & ppm & 9 & 7 \\
\hline $\mathrm{Zn}$ & ppm & 1.9 & 2.1 \\
\hline $\mathrm{Cu}$ & ppm & 5.7 & 6.2 \\
\hline $\mathrm{Fe}$ & ppm & 16 & 13 \\
\hline Mn & ppm & 4.3 & 4.3 \\
\hline B & ppm & 0.58 & 0.32 \\
\hline$\sum$ Bases & & 22.65 & 16.11 \\
\hline
\end{tabular}

Fuente: Análisis químicos de suelo Elaborado: por los autores

En la figura 2, se observa la comparación del contenido nutricional del suelo, con respecto a la materia orgánica existe un incremento conforme va creciendo la plantación el desarrollo de materia orgánica es mayor, esto se podría deber a la generación de materia orgánica por parte de poda y hojarascas de las plántulas de cacao como de procesos de limpieza de malezas como lo indica (Barrezueta \& Paz, 2017). Los macronutrientes Nitrógeno $(\mathrm{N})$ y Potasio $(\mathrm{K})$ presentan un ligero descenso lo contrario al Fosforo $(\mathrm{P})$, los mesonutrientes Calcio ( $\mathrm{Ca}$ ), Magnesio $(\mathrm{Mg})$ y Azufre se encuentran en menores cantidades, los micronutrientes Zinc $(\mathrm{Zn})$ y Cobre $(\mathrm{Cu})$ presentan un aumento, diferente al Hierro (Fe) y Boro (B) que a los 2 años de las plántulas ha disminuido las concentraciones de estos elementos en el suelo, con respecto al Manganeso (Mn) la concentración no ha variado.

Los niveles medios y bajos de nutrientes en el suelo del lote al momento de realización del análisis químico de suelo a los 2 años de las plántulas de cacao se deben, a que la cantidad de fertilizantes que anualmente se le aplica, no han sido suficientes para construir un almacén duradero de estos elementos en el suelo (Villalba Martínez et al., 2020). 
La capacidad de intercambio de cationes corresponde a la sumatoria de los meq/100mL de $\mathrm{Ca}, \mathrm{Mg}, \mathrm{K}$ y acidez extraíble, un valor inferior a 5 se considera bajo; entre más alto sea el índice mayor capacidad de nutrición tendrá el suelo de acuerdo con (Navarro et al., 2011). El control previo a la plantación de las plántulas se encuentra con un valor de 22.65, tiene una cantidad adecuada de iones, y el control a los 2 años de plantación presentó un valor de 16.11 de manera que se encuentra por debajo del control previo a la plantación, pero sigue siendo adecuado.

\section{Figura 2}

Comparación del contenido nutricional del suelo previo a la plantación de cacao \& 2 años de cultivo

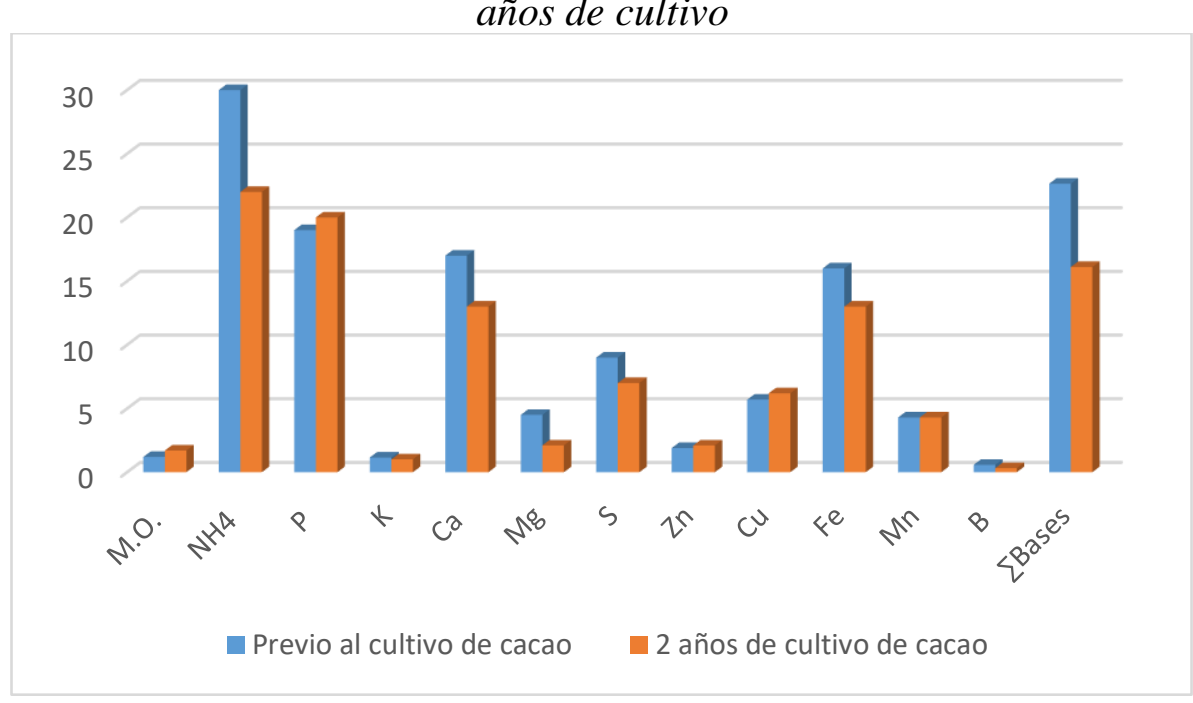

Fuente: Análisis químicos de suelo

Elaborado: por los autores

De acuerdo con (Bertsch, 1987) a respecto de las relaciones catiónicas, el contenido mayor de cationes en el suelo ( $\mathrm{Ca}, \mathrm{Mg}$ y $\mathrm{K}$ ) puede estar en cantidades altas, sin embargo, existe la posibilidad que la absorción de los mismos por la planta sea mala, debido a que se encuentran en proporciones desequilibradas. Con respecto a esto se complementó el análisis químico del suelo realizado a los 2 años de la plantación de cacao, el resultado obtenido para el control en la relación catiónica $\mathrm{Ca} / \mathrm{Mg}$ es de 6.1 por lo tanto esta en desbalance, ya que el intervalo de balance se encuentra entre 2 a 5 . En la relación $\mathrm{Mg} / \mathrm{K}$ es de 2.08, el cual es un poco bajo con respecto al intervalo de balance que va de 2,5 a 15. Para la relación catiónica $(\mathrm{Ca}+\mathrm{Mg}) / \mathrm{K}$ el intervalo se encuentra entre 10 y 40, el lote presentó un valor de 14.95 de tal manera se encuentra dentro del balance de esta relación catiónica, los datos de los valores de las relaciones de balance se obtuvieron de (Navarro et al., 2011). De las relaciones catiónicas evaluadas solo la relación $(\mathrm{Ca}+\mathrm{Mg}) / \mathrm{K}$ se encuentra balanceada.

Haciendo énfasis en la relación del $\mathrm{pH}$ con la disponibilidad de elementos por las plántulas de cacao del análisis químico del suelo a los 2 años de plantación y como es natural el $\mathrm{pH}$ en suelos alcalinos se relaciona positivamente con la presencia elevada de calcio hace que el fósforo que contiene un suelo reaccione con él, formando fosfatos cálcicos insolubles, y, por lo tanto, no aprovechables por las plantas (Catalán, 2016), esto 
explicaría las altas concentraciones de calcio encontradas en el análisis y el aumento de niveles de fosforo de la comparación de los análisis, también se observa una relación positiva del $\mathrm{pH}$ con magnesio y potasio, estos aumentan a medida que aumenta el $\mathrm{pH}$ (Borges et al., 2012), con los microelementos hierro, manganeso y boro correlacionaron negativamente con el aumento del $\mathrm{pH}$, esto se debe a que los micronutrientes son mejores absorbidos en suelos ácidos y la disponibilidad de materia orgánica (Roca et al., 2007).

La materia orgánica aumenta la disponibilidad de micronutrientes, pero tiene mejor influencia en zinc y cobre (Villalba Martínez et al., 2020), esto explicaría el aumento de estos micronutrientes en el análisis de los 2 años y en comparación con el análisis previo se observa una relación positiva de la materia orgánica y estos micronutrientes, de igual forma la fertilización con macronutrientes incrementa la disponibilidad de micronutrientes en el suelo (Zhang, Wang \& Jin, 2001; Li et al., 2007).

\section{Conclusiones.}

- Los resultados de los parámetros del análisis químico del suelo presentan variaciones al compararlos, existe una tendencia en la disminución de nutrientes en el suelo mientras avanza el crecimiento de las plántulas de cacao.

- Según los resultados del muestreo, el sistema inicial productivo presenta desbalances nutricionales y deficiencias en contenidos de nutrientes para su desarrollo, por lo cual se requiere de planes de fertilización que garanticen una adecuada sostenibilidad del sistema.

- El pH que tiene el suelo según el último análisis es ligeramente alcalino el cual se relaciona directamente con los altos contenidos de calcio, magnesio, potasio e incremento de fosforo, así como la deficiencia de micronutrientes, para corregirlo debe de contemplarse la adición de acidificantes según recomendaciones técnicas ajustadas al caso.

- El lote presenta bajo contenido de materia orgánica, por ende, la obtención de nutrientes a partir de la mineralización es poca, el aumento de las concentraciones de materia orgánica en el suelo eleva los niveles nutricionales de estos según la actividad biológica que este contenga, así mismo permite su acidificación, es necesario contemplar la adición de abonos orgánicos conjunto con una correcta humedad del suelo, hasta que la plantación de cacao con sus hojarascas y material vegetativo de poda con el paso de los años, permita la autoproporción de materia orgánica.

\section{Referencias bibliográficas.}

Amésquita, E., Barrera, L., Burbano, H., Clavijo, J., Espinosa, J. A., García, A., Guerrero, R., León, A., Lora, R., Malavolta, E., Muñoz, R., Villar, H., \& Zapata, R. (2001). Fertilidad de Suelos. Diagnóstico y Control. In Sociedad Colombiana de la Ciencia del Suelos. 
Arvelo Sánchez, M. A., González León, D., Maroto Arce, S., Delgado López, T., \& Montoya López, P. (2017). Manual técnico del cultivo de cacao Buenas prácticas para América Latina. In Instituto Interamericano de Cooperación para la Agricultura (IICA). file://C:/Users/usuario/Downloads/BVE17089191e.pdf

Barrezueta, S., \& Paz, A. (2017). Estudio comparativo de la estructura elemental de materia orgánica de suelo y mantillo cultivados de cacao en El Oro, Ecuador. Revista Científica Agroecosistemas, 5(January), 54-62. https://aes.ucf.edu.cu/index.php/aes/article/view/141

Bertsch, F. (1987). Manual Para Interpretar la Fertilidad de Los Suelos de Costa Rica Floria Bertsch - Google Libros (2nd ed.). Editorial Universidad de Costa Rica. https://books.google.es/books?hl=es\&lr=\&id=tJz0G2oLs5gC\&oi=fnd\&pg=PA1\&d $\mathrm{q}=$ cuadros+de+analisis+de+fertilidad+optima+de+suelos\&ots=36GqBdaqip\&sig= cBDTOY91z_hAsjaIgJLdqWXLw4E\#v=onepage\&q=cuadros de analisis de fertilidad optima de suelos\&f=false

Borges, J. A., Barrios, M., Sandoval, E., Bastardo, Y., \& Márquez, O. (2012). Características físico-químicas del suelo y su asociación con macroelementos en áreas destinadas a pastoreo en el estado Yaracuy. Bioagro, 24(2), 121-126. http://ve.scielo.org/scielo.php?script=sci_arttext\&pid=S1316$33612012000200006 \& \operatorname{lng}=\mathrm{es} \& n r m=i s o \& \operatorname{lng}=\mathrm{en}$

Catalán, G. (2016). El pH del suelo en la agricultura. Agropal. http://www.agropal.com/es/el-ph-del-suelo/

Lamz, A., \& González, M. C. (2010). EFECTO DEL ESTRÉS POR NaCl EN EL CRECIMIENTO Y LAS RELACIONES HÍDRICAS EN PLANTAS DE TOMATE (Solanum lycopersicum L.) DURANTE EL PERÍODO VEGETATIVO. Cultivos Tropicales, 31(4), 76-81.

Ministerio de Agricultura y Ganadería. (2014). Resultados nacionales sobre el III Censo Nacional Agropecuario de la República del Ecuador. In Censo Nacional Agropecuario (Vol. 1). https://www.ecuadorencifras.gob.ec/documentos/webinec/Estadisticas_agropecuarias/CNA/Tomo_CNA.pdf

Navarro, D., Milagro, R., \& Ruíz, P. (2011). Análisis de fertilidad de suelos en el laboratorio de Química del Recinto de Grecia, Sede de Occidente, Universidad de Costa Rica Soil fertility analysis in the laboratory of chemical campus - Grecia, Sede de Occidente, Universidad de Costa Rica. InterSedes, XII(23), 23. https://revistas.ucr.ac.cr/index.php/intersedes/article/view/980

Osorio, N. W. (2012). pH DEL SUELO Y DISPONIBILIDAD DE NUTRIENTES. Manejo Integral Del Suelo y Nutrición Vegetal, 1(4), 4-7. http://www.walterosorio.net/web/sites/default/files/documentos/pdf/1 $4 \mathrm{pH}$ del suelo y nutrientes_0.pdf 
Pinargote, M. (2010). Comportamiento productivo de cacao (Theobroma cacao L.) CCN51 ante diferentes formulaciones de fertilización. UNIVERSIDAD TÉCNICA ESTATAL DE QUEVEDO.

Quiroz, J., \& Mestanza, S. (2012). Establecimiento y manejo de una plantación de cacao. In Programa Nacional del Cacao (Issue 146).

Roca, N., Pazos, M. S., \& Bech, J. (2007). Disponibilidd de cobre, hierro, manganeso, zinc en suelos del no Argentino. Ciencia Del Suelo, 25(1), 31-42.

Sosa, D. A. (2012). Técnicas de toma y remisión de muestras de suelos. In Instituo Nacional de Tecnol igía Agropecuaria.

Villalba Martínez, C. J., Merino García, A., \& Etchevers Barra, J. (2020). Diagnóstico de la fertilidad química de suelos (Rhodic Paleudult) en sistemas agrarios y forestal de la región Oriental de Paraguay. 1 Universidad Nacional de Caaguazú. Coronel Oviedo, Paraguay. 2 Universidad Santiago de Compostela. Santiago de Compostela, España. 3 Colegio de Postgraduados, Campus Montecillo. Montecillo, México., 22(2), 92-99.

Zhang, S.X., Wang, X.B. \& Jin, K. (2001). Effect of different N and P levels on availability of zinc, copper, manganese and iron under arid conditions. Plant Nutr. Fert. Sci. 7, 391-396.

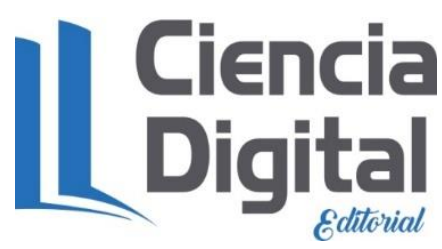




\section{PARA CITAR EL ARTÍCULO INDEXADO.}

Briones García, J. I., Mejía Reinoso, T. J., \& Briones García, H. D. (2021). Evaluación de la fertilidad química del suelo en un sistema inicial productivo de Theobroma cacao de la finca "Hermanos Briones", Portoviejo - Ecuador . ConcienciaDigital, 4(3.1), 227237. https://doi.org/10.33262/concienciadigital.v4i3.1.1825

\section{¿Ciencia}

El artículo que se publica es de exclusiva responsabilidad de los autores y no necesariamente reflejan el pensamiento de la Revista Conciencia Digital.

El artículo queda en propiedad de la revista y, por tanto, su publicación parcial y/o total en otro medio tiene que ser autorizado por el director de la Revista Conciencia Digital.

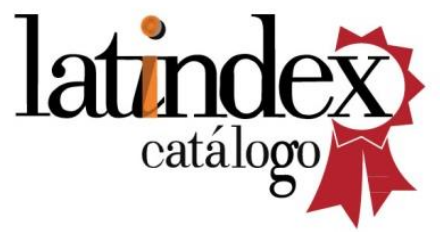

